\title{
Correction: IL-24 deficiency protects mice against bleomycin- induced pulmonary fibrosis by repressing IL-4-induced M2 program in macrophages
}

Li-Zong Rao · Yi Wang • Lei Zhang · Guorao Wu • Lu Zhang • Fa-Xi Wang • Long-Min Chen · Fei Sun · Song Jia •

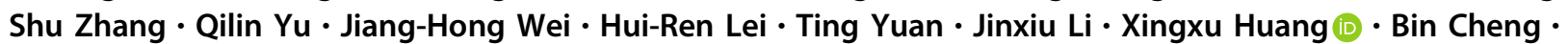
Jianping Zhao $\cdot$ Yongjian Xu $\mathrm{Xi}$-Wen Mo $\cdot$ Cong-Yi Wang $(\mathbb{B} \cdot$ Huilan Zhang

Published online: 5 January 2021

(c) The Author(s), under exclusive licence to ADMC Associazione Differenziamento e Morte Cellulare 2021

Correction to: Cell Death \& Differentiation

https://doi.org/10.1038/s41418-020-00650-6
The original version of this article unfortunately contained a mistake. In the "Acknowledgments" part, the funding number "9174927" (in the second line) should be corrected to "91749207". The original article has been corrected. 